\title{
Biphasic fluid oscillator with coaxial injection and upstream mass and momentum transfer
}

\author{
M. Heuberger ${ }^{1}$ L. Gottardo ${ }^{1}$ M. Dressler ${ }^{2,3} \cdot$ R. Hufenus ${ }^{1}$
}

Received: 26 January 2015 / Accepted: 27 April 2015 / Published online: 19 May 2015

(C) The Author(s) 2015. This article is published with open access at Springerlink.com

\begin{abstract}
We present model experiments with biphasic flow and computational fluid dynamics (CFD) simulations for concentric co-flow scenarios. A lower viscosity fluid is injected into an outer phase of reduced thickness. Static design modifications of the injection geometry are then studied to allow for self-adjusting upstream transfer of mass and momentum. Such static arrangement gives rise to a simple biphasic fluid oscillator that can produce individual droplets at high rates and high aspect ratios. Frequency analysis and CFD simulations are invoked to shed light on the physics of this dynamically forced jet breakup and to identify relevant control parameters. In addition, we illustrate how a terminal baffle plate at the nozzle can produce a split-up into multiple dripping or jetting threads depending on its relative rotational symmetry with the upstream mass transfer. The here-presented distinctive injection geometry bears potential for simple ways of controlled jet breakup in microfluidics devices, which currently primarily rely on Rayleigh-Taylor breakup or the costly introduction of intricate actuators or compliant elements. Most notably, this oscillatory injector has potential for application in biphasic melt-flow spinnerets to realize advanced fiber core structures during melt-spinning.
\end{abstract}

M. Heuberger

manfred.heuberger@empa.ch

1 Laboratory for Advanced Fibers, Empa, Swiss Federal Laboratories for Materials Science and Technology, St. Gallen, Switzerland

2 Department of Chemical Engineering, University of Massachusetts Amherst, Amherst, MA, USA

3 Present Address: Exa Corporation, Burlington, MA, USA
Keywords Microfluidic oscillator - Droplet formation · Co-flow $\cdot$ Confinement $\cdot$ Jetting $\cdot$ Injection geometry Melt-spinning

\section{Introduction}

The scientific interest in the physics of co-flowing liquids has started far more than a century ago (Plateau 1873; Rayleigh 1879). The stability of the inner jet and its transition to dripping has since received a considerable amount of attention (Eggers and Villermaux 2008), particularly with the advent of microfluidic devices where controlled droplet formation (Rosenfeld et al. 2014) has become key for promising applications involving chemical droplet reactors (Nightingale et al. 2014) or biological single-cell analysis (Rakszewska et al. 2014). The interest in the stability of coflow also comes from various existing industrial processes related to materials processing. In a prominent example, co-flow is used to efficiently pump highly viscous oil inside a pipe, by the use of lower viscosity water, which is pumped alongside and therefore acts as a lubricant toward the wall (Bannwart 2001). The topological stability of the multiphase fluid flow has to be assured in this case; understanding and control of biphasic co-flows are thus relevant for fluid conveying (Joseph et al. 1997; Ghosh et al. 2009) or pumping through transport lines (Hooper and Boyd 1983).

Our interest in this model study of biphasic co-flow originates in yet another application area, which is the combination of a polymer melt and a liquid at elevated temperatures to obtain a structured fluid core fiber template for subsequent solidification by draw-cooling. Control of the fluid core structure in a fiber is wanted to adjust fluid storage, transport or rheological damping properties. During 
bicomponent fiber melt-spinning, the polymer melt and a fluid are concentrically merged inside the spinneret using a static steel microfluidic nozzle. Melt-spun fibers with a fluid core can exhibit a range of interesting transport or dissipation properties depending on the choice of the fluid rheology and the solidified core topology.

Known strategies to realize droplet formation or oscillatory flow in microfluidics include squeeze-off in flowfocusing devices (Gupta et al. 2014), oscillatory switching with compliant diaphragms (Mosadegh et al. 2010; Xia et al. 2010) or pneumatics (Nguyen et al. 2012) or the use of external capacitance to realize an oscillator with a remarkable kinetic tuning range (Kim et al. 2013). One of the technologically most advanced examples is a collection of digital fluid logics, NAND and NOR gates, that can be cascaded into a logic oscillator (Devaraju and Unger 2012). In this work, we present a simple and passive adaptation of the nozzle geometry that adds upstream self-adjusting transfer of momentum and mass to excite high-frequency modes of oscillatory jet breakup into droplets.

The underlying physics of liquid jets is reviewed by Eggers and Villermaux (Eggers and Villermaux 2008), where it is emphasized how important is the interfacial tension, which also defines the jet-relevant dimensionless Weber, capillary and Ohnesorge numbers.

Historically, the first-documented systematic study analyzing jet dynamics was performed by Lord Rayleigh (1879), hence the so-called Rayleigh (linear) stability analysis, which essentially states that instabilities with wavelengths larger than the jet diameter will grow in amplitude. The often-cited Rayleigh-Plateau (Plateau 1873; Rayleigh 1879) instability model thus predicts jet breakup driven by perturbation and interfacial tension-exhibiting a characteristic wavelength of maximum growth rate. Topologies of co-annular flow were further investigated by Tomotika (1935), (Meister and Scheele 1967, 1969a, b; Scheele and Meister 1968) who studied and described the general behavior of a jet in a stationary surrounding medium. Cramer et al. (2004) experimentally investigated droplet formation at a capillary tip. This work allowed distinction between dripping at the nozzle and jetting with later instability breakup. Increasing the velocity of the continuous outer phase and lowering the interfacial tension provoked a decrease in the droplet size. Utada et al. (2007) studied the dripping-to-jetting transition experimentally for a liquid passing through an orifice into an immiscible fluid. They suggested that this transition can take place via two different mechanisms as summarized in a state diagram where the dripping-to-jetting transition is discussed on the footings of outer capillary number and inner Weber number.

The direct numerical simulation of jet dynamics is a valuable additional tool to study readily observable phenomena like jet breakup (Richards et al. 1994; Utada et al.
2008), droplet dimensions (Cramer et al. 2004) or dynamics of droplets formation (Richards et al. 1995). Notably, the work of Richards et al. (1994) focused on studying the axisymmetric, dynamic breakup of a Newtonian liquid jet at various Reynolds numbers ( $\mathrm{Re}$ ), injected vertically into another immiscible Newtonian liquid. They included numerical simulations using an algorithm based on the volume of fluid (VOF) method, to model the transition from a jet start-up to the breakup into droplets.

Here we describe the effect of a new element, which is upstream momentum and mass transfer, to excite a dynamic biphasic fluid oscillation in two immiscible model fluids. Crucial features of the observed flow topologies are reproduced in the framework of CFD simulations, which also provide useful additional insights into the workings of this fluid oscillator.

\section{Materials and methods}

\subsection{Co-flow apparatus}

To visualize the liquid-liquid interface as described in this paper, we built the experimental setup operated at low Reynolds numbers ( $\operatorname{Re} \sim 0.01-1)$ as shown in Fig. 1. Fluids are stored in two glass containers of volume $500 \mathrm{ml}$ each. The gas-spring pressurization inside the containers can be controlled in a range from 0.02 to 2 bar using pressure reducers (SMC, IR2000 F02; NORGREN, 11-818999) that are fed from a 6 bar pressurized air supply. The fluids are then pressed through flexible tubes in a continuous flow to enter our model liquid-liquid injection geometry consisting of an outer glass tube and a coaxially centered inner stainless steel capillary (Hamilton, G11). The capillary inner diameter is $D_{\mathrm{i}}=2.39 \mathrm{~mm}$, and the outer diameter is $D_{\text {on }}=3.05 \mathrm{~mm}$; the total length of the setup is $L_{1}=100 \mathrm{~mm}$. The inner diameter of the glass tube is $D_{\mathrm{o}}=4 \mathrm{~mm}$, and its length is $L_{2}=60 \mathrm{~mm}$. The inner capillary is extending into the glass tube over a length of $L_{\mathrm{n}}=20 \mathrm{~mm}$. The setup is built into a Swagelok "Tee" shape connection (SS-10M0-3-10M-6M).

We used two types of injection capillaries: a standard one, labeled N0, as well as a capillary with four side openings, labeled $\mathrm{N} 4-$ the cipher indicating the number of side openings. The side openings have a height of $H=2.3 \mathrm{~mm}$, a width of $W=0.8 \mathrm{~mm}$, and the upper and the lower ends of the openings are rounded (cf. Fig. 2). The distance between the openings mid-height and the tip of the capillary is fixed at $D_{\mathrm{mhn}}=6.2 \mathrm{~mm}$. The openings are equally distributed around the circumference of the capillary, i.e., every $90^{\circ}$ for the N4 capillary. Schematics of the capillaries, along with their physical dimensions, are shown in Fig. 2. 

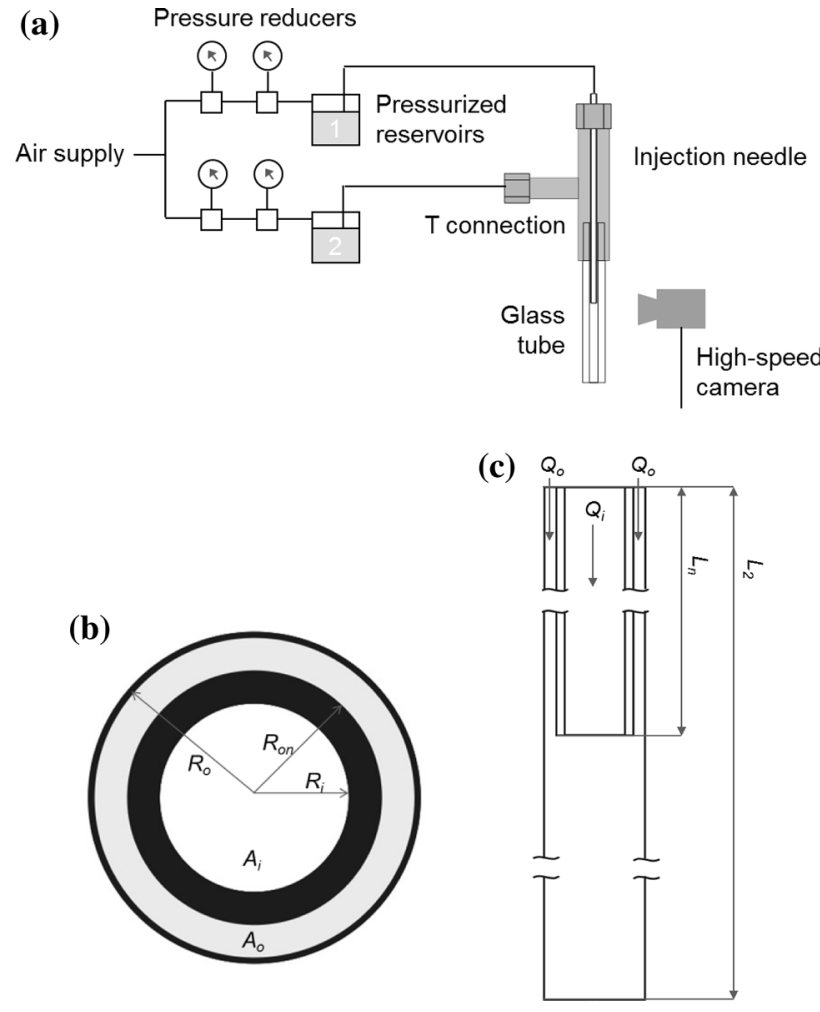

Fig. 1 a Experimental setup for co-flow experiments with liquid containers, Tee-connector and glass tube. b Cross section details at the co-flow nozzle defining inner radius of injection capillary $R_{\mathrm{i}}$, outer radius of injection capillary $R_{\mathrm{on}}$, radius of outer tube $R_{\mathrm{o}}$, and the nominal areas occupied by the inner and outer fluid, $A_{\mathrm{i}}, A_{\mathrm{o}}$. c Longitudinal section of the co-flow nozzle. $Q_{\mathrm{i}}, Q_{\mathrm{o}}$ are the flow rates of the inner and the outer fluid at the inlet, respectively

An optional baffle plate could be attached in form of a final cap to the capillary to induce variable flow splittings. The resulting flow patterns shall be discussed at the end of the paper.

A high-speed camera (Casio EXF1, $300 \mathrm{fps}$ ) was used to record movies of the flow patterns.

Flow rates of the different fluids were calibrated gravimetrically against applied pressure. We note that this experimental setup with air supply, pressure reducers and pressurized reservoirs can lead to a backflow of the inner phase when the driving pressures of the two phases differ too much. This limitation was accepted intentionally because it replicates the conditions at the fiber melt-spinning process, which shows similar pressure-driven characteristics.

We note, however, that neither pressure driving nor backflow is taken into account in the CFD simulations used here, since global mass conservation is inherent to the flow simulations by setting the divergence of the velocity field to zero (Eq. 2). This computational approach proved to be sufficient because we did not experimentally explore the backflow regime.

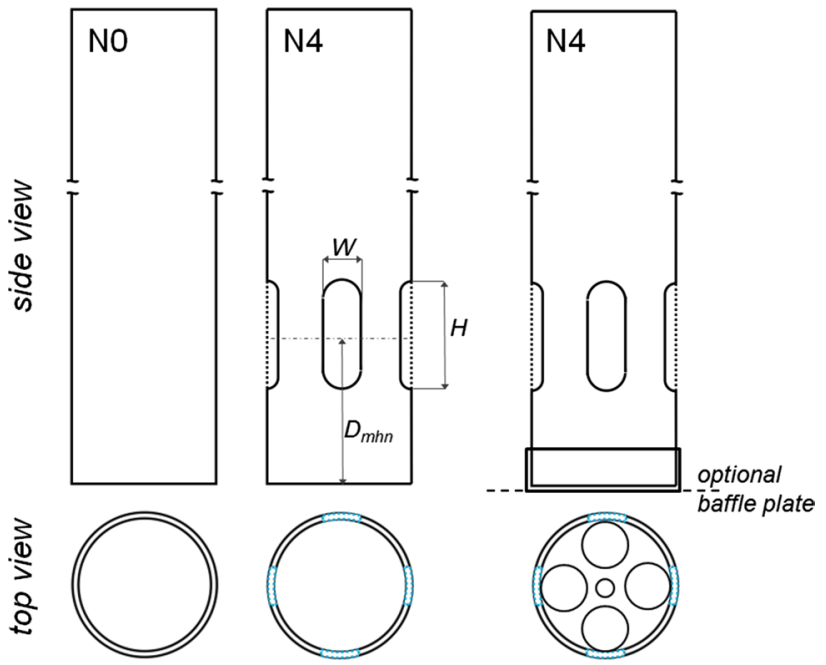

Fig. 2 Schematics of the standard injection capillary (N0) and the alternative injection capillary with four side openings (N4). The side openings with height $H=2.3 \mathrm{~mm}$ and width $W=0.8 \mathrm{~mm}$ are equally distributed around the circumference of the capillary. The distance between openings center and tip is $D_{\text {mhn }}=6.2 \mathrm{~mm}$. (Right) Optionally, we caped the capillary with a baffle plate; shown here is a baffle plate with five circular openings, four of which are oriented $0^{\circ}$, i.e., in registry with the upstreams capillary side openings

It is noteworthy that for our experimental setup, the injection ratio $\sqrt{\frac{A_{\mathrm{i}}}{A_{\mathrm{o}}}}=0.78$ is larger than the ratios typically used in most previous experiments (Meister and Scheele 1967, 1969a, b; Cramer et al. 2004; Utada et al. 2007; CastroHernández et al. 2009) and in modeling (Vempati et al. 2007, 2010) or in related simulation work (Kouris and Tsamopoulos 2001, 2002), where the injection ratio had been between 0.002 and 0.02 . The injection ratios of the flow geometries studied previously were so small that the flows could be considered as fully developed bulk flows that are notably not affected by confinement effects (Guillot et al. 2007; Nunes et al. 2013); yet confinement is relevant for our experiment, as we shall see.

\subsection{Computational fluid dynamics (CFD)}

For a description of Newtonian flow of incompressible multiphase fluids, the appropriate physical variables are the scalar volume fraction function, $F$, and the averaged velocity vector field $\mathbf{v}$. The scalar volume fraction function describes the variation of density and viscosity across the moving liquid/liquid interface, i.e., $x=F x_{2}+(1-F) x_{1}, x \in\{\rho, \eta\}$. Subscripts 1 and 2 denote the fluid property of inner and outer phase, respectively. The dynamic equations for these two variables read (Vempati et al. 2007, 2010):

$\frac{\partial F}{\partial t}=-\boldsymbol{v} \cdot \nabla F$, 
Table 1 Fluid parameters $\left(25^{\circ} \mathrm{C}\right)$

\begin{tabular}{lllll}
\hline & Density $\rho\left(\mathrm{g} / \mathrm{cm}^{3}\right)$ & Viscosity $\eta(\mathrm{mPas})$ & $\begin{array}{l}\text { Surface tension } \gamma \\
(\mathrm{mN} / \mathrm{m})\end{array}$ & Flow rates Q $(\mathrm{ml} / \mathrm{s})$ \\
\hline Water (inner) & 1 & 1 & 72 & $1.2-5$ \\
Vaseline oil (outer) & 0.81 & 170 & 40 & $0.062-1$ \\
Fluid-fluid interface & & & $50^{\mathrm{a}}$ & \\
\hline
\end{tabular}

${ }^{\text {a }}$ Estimation was varied $\pm 15 \mathrm{mN} / \mathrm{m}$ in CFD simulations $\nabla \cdot \boldsymbol{v}=0$

$\rho \frac{\partial \boldsymbol{v}}{\partial t}=-\rho \boldsymbol{v} \cdot \nabla v-\nabla p+\nabla \boldsymbol{\sigma}+\gamma \kappa \nabla F$

Equation (1) is the time evolution equation of the volume fraction function $F$ with the local derivative of the function $F$ on the left-hand side and the negative convective derivative of $F$ on the right-hand side. Equation (1) describes advection of the fluid interface through the flow. Equation (2) is the continuity equation for an incompressible fluid, i.e., the divergence-free property of the velocity. Equation (3) is the momentum balance equation of the fluid where $\rho$ is the effective mass density, $p$ is the pressure, $\boldsymbol{\sigma}=\mu\left(\nabla v+\nabla v^{T}\right)$ is the Newtonian stress tensor, $\gamma$ is the interfacial tension, and $\kappa$ is the curvature of the liquid/liquid interface. The curvature of the fluid interface is given as the divergence of the normal vector pointing from phase 1 into phase 2 , i.e.,

$\kappa=-\nabla \cdot \hat{\mathbf{n}}=\frac{1}{|\mathbf{n}|}\left[\left(\frac{\mathbf{n}}{|\mathbf{n}|} \cdot \nabla\right)|\mathbf{n}|-(\nabla \cdot \mathbf{n})\right]$,

where $\hat{\mathbf{n}}=\mathbf{n} /|\mathbf{n}|$ is the local normal unit vector on the interface and the normal vector on the interface and $\mathbf{n}$ is given as the gradient of the interfacial volume fraction function $\mathbf{n}=\nabla F$.

Equations (1-4) have been used in the past to simulate the injection of a Newtonian liquid into another stationary Newtonian fluid. In these studies, the focus was on specific aspects of this flow problem, e.g., jetting in the high Reynolds number regime (Richards et al. 1993), breakup of moving jets in droplets far away from the injection capillary (Richards et al. 1994) or the competition of drop and jet formation (Richards et al. 1995). In all of these studies (Richards et al. 1993, 1994, 1995), the outer phase is kept stationary and a standard injection capillary is considered. Furthermore, in reference (Richards et al. 1994), the viscosity ratio is about one order of magnitude larger than in the present study, and the density ratio is smaller than unity, whereas in the present study it is larger than unity.

We have been able to reproduce the results for the stationary outer phase flow using the interFoam solver of the OpenFOAM multiphysics package.
The interFoam solver has been exploited and adopted for various engineering applications in the past, e.g., (Deshpande et al. 2012; Higuera 2013a, b). Furthermore, OpenFOAM was used to solve the flow of water and Vaseline through the non-standard flow geometry which involves an injection capillary with four lateral side openings and the co-flowing outer medium.

\subsection{Model fluids used}

The fluids are chosen to mimic injection of an immiscible fluid into a polymer melt, with the simplification that nonlinear viscosity effects (e.g., viscoelasticity) of the melt are not represented here. We thus used water as injected phase and Vaseline oil (GPR Rectapur, VWR) as outer phase. We realize that room-temperature Vaseline does not accurately model the complex viscoelastic behavior of a polymer melt. Some of the relevant fluid properties are summarized in Table 1. All experiments have been performed at room temperature $\left(25^{\circ} \mathrm{C}\right)$.

In the experiments, the inner flow rate, $Q_{\mathrm{i}}$, varied over a maximum range of $1.2-4.2 \mathrm{ml} / \mathrm{s}$, and the outer flow rate, $Q_{\mathrm{o}}$, was varied within the range of 0.062 and $1 \mathrm{ml} / \mathrm{s}$.

We label the injected inner phase with subscript "i" and the continuous outer phase with subscript "o". The physical parameters are hence described using the symbols $\rho_{j}, \eta_{j}, \gamma$, $Q_{j}, v_{j}$ denoting density, viscosity (kinematic and dynamic), fluid-fluid interface tension, volumetric flow rate and nominal flow velocity with subscript $j=\mathrm{i}$,o differentiating inner and outer phase.

\section{Results and discussion}

\subsection{Co-flow regimes}

We conducted fluid flow experiments with two different injection capillaries inside a glass tube. Besides a plain capillary, we also used injection capillaries with four upstream side openings as shown in Fig. 2. We refer to them as injection geometries N0 and N4, respectively. We can readily differentiate between two different regimes of co-flow topologies, namely dripping and jetting. While individual droplets are leaving the capillary tip in the former, the 
jetting regime produces a continuous fluid core, at first. It is empirically convenient to subdivide this latter jetting regime into three sub-regimes-accounting for subsequent breakup behavior and lateral jet stability. Throughout this discussion, we thus adopt a terminology referring to four co-flow topology classes:

- Dripping or drops (D): separated droplets are generated at the nozzle, and these droplets are entrained downstream; notably, we will show that the N4 geometry introduces topological oscillations inside the nozzle, which can give rise to a dynamically driven dripping mechanism as opposed to the well-known interface tension-driven Rayleigh-Plateau instability seen with the N0 geometry.

- Slugs (S): continuous jetting at the capillary tip, followed by Rayleigh-Plateau instability with downstream breakup into oblong slugs.

- Core annular (CA): continuous jetting regime without breakup by Rayleigh-Plateau instability - the jet leaves the observation tube before breaking up.

- Core annular undulant (CAU): jetting regime characterized by perturbations produced at the nozzle consisting of wavelengths that are weakly growing while propagating downstream and can be substituted by faster growing, longer wavelengths Rayleigh-Plateau instabilitiesthe jet thus appears to undulate irregularly and it leaves the observation tube before completed breakup.

For the reader's convenience, Fig. 3 illustrates four representative snapshots of these four co-flow topology classes. It is noted that biphasic fluid drainage occurs at the end of our transparent glass tube, which acts as a
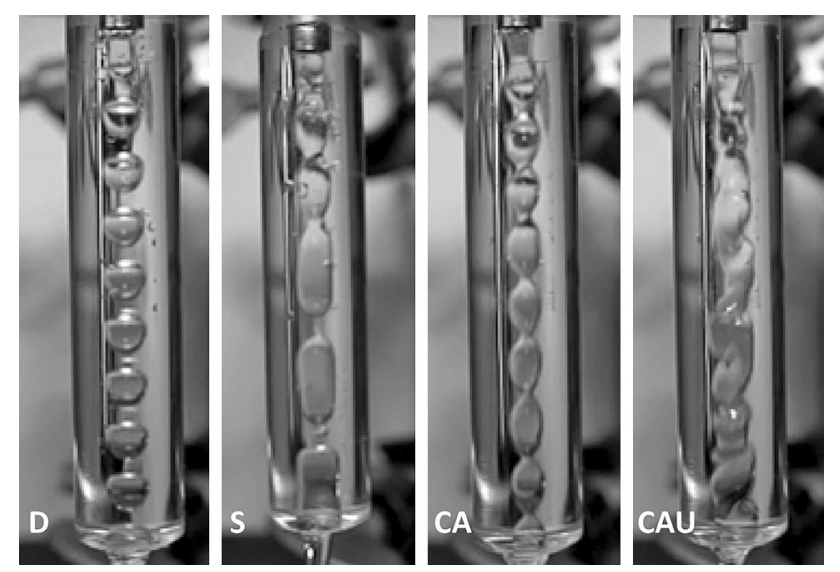

Fig. 3 Classes of typical co-flow topologies seen in our view tube, the co-flow nozzle is at the top of each image: dripping (D) $\left(Q_{\mathrm{i}}=1.75 \mathrm{ml} / \mathrm{s} ; Q_{\mathrm{o}}=0.49 \mathrm{ml} / \mathrm{s}\right) ; \operatorname{slugs}(\mathrm{S})\left(Q_{\mathrm{i}}=1.20 \mathrm{ml} / \mathrm{s}\right.$; $\left.Q_{\mathrm{o}}=0.22 \mathrm{ml} / \mathrm{s}\right)$; core annular $(\mathrm{CA})\left(Q_{\mathrm{i}}=2.22 \mathrm{ml} / \mathrm{s}, Q_{\mathrm{o}}=0.49 \mathrm{ml} / \mathrm{s}\right)$; core annular undulant (CAU) $\left(Q_{\mathrm{i}}=3.17 \mathrm{ml} / \mathrm{s} ; Q_{\mathrm{o}}=0.49 \mathrm{ml} / \mathrm{s}\right)$ nozzle into air at this point (see bottom Fig. 3), where it can, once more, produce dripping or jetting into air. Indeed, at a sufficiently small capillary number of the outer fluid, $C a_{\text {o }} \leq 0.10$, we observed a minor back feed modulation of the inner jet breakup (i.e., slug length) due to flow variations induced by dripping at the end of the view tube; however, the effect remains small and has a measurable effect on topology only in the lower half of the glass tube; it is to be neglected in the following discussion.

To further assess the underlying fluid jet physics, it is useful to present the different co-flow topologies in a state diagram as proposed by previous work (Utada et al. 2007; Nunes et al. 2013). The diagram is displayed separately for the two injection geometries in Fig. 4a, b, respectively. For
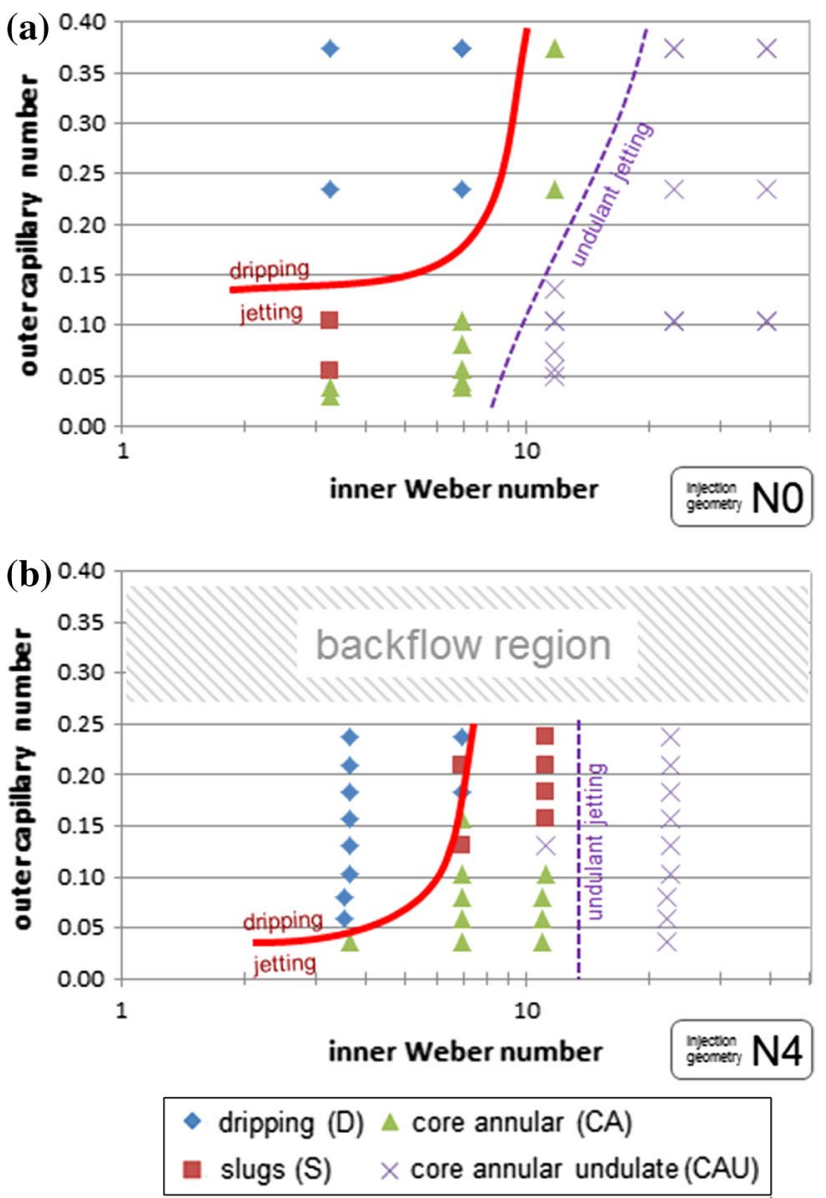

Fig. 4 State diagram of interface topologies observed with both injection geometries, a N0 and b N4, as a function of dimensionless outer capillary number $C a_{\mathrm{o}}$ and inner Weber number, $W e_{\mathrm{i}}$ (log scale). The dripping-to-jetting transition and the transition to undulant flow topologies at higher inner Weber numbers $W e_{\mathrm{i}} \geq 10$ are marked by lines. The $\mathrm{N} 4$ and $\mathrm{N} 0$ injection geometries are experimentally limited to $C a_{\mathrm{o}} \leq 0.25$ and $C a_{\mathrm{o}} \leq 0.40$, respectively, due to inner phase backflow. We think that the backflow limitation is introduced by the gas-spring pressurization of the fluids in the reservoirs as described in Sect. 2.1; our experimental setup adds external gas-spring compliance to the fluids 
the vertical axis, we plot the dimensionless capillary number of the outer fluid, $C a_{\mathrm{o}}=\eta_{0} \mathrm{v}_{0} / \gamma$, and for the horizontal axis, we plot the dimensionless Weber number of the inner fluid, $W e_{\mathrm{i}}=\rho_{\mathrm{i}} \mathrm{v}_{\mathrm{i}}^{2} \Lambda / \gamma$, with the droplet diameter, $\Lambda$. The outer capillary number describes the ratio of outer fluid momentum to interfacial tension. At $C a_{\mathrm{o}} \gg 1$, the outer fluid momentum will dynamically bend the interfacial topology. The inner Weber number is the ratio of jet kinetic energy to interface tension. At $W e_{\mathrm{i}} \gg 1$, the jet kinetics outweighs its interfacial energy and therefore jetting predominates (Eggers and Villermaux 2008; Nunes et al. 2013).

First of all we note in Fig. 4 that all our real fluid experiments were conducted at $W e_{\mathrm{i}}>1$. It is thus not surprising that we observe inner nozzle jetting in all cases except for class " $\mathrm{D}$ " at $W e_{\mathrm{i}} \leq 7$. The dripping-to-jetting transition is conveniently marked by a plain line in the state diagram (Fig. 4). If we first consider the diagram obtained with the N0 injection geometry, there is qualitative and close quantitative agreement to the dripping-to-jetting transition with previous work by Utada et al. (2007, 2008). However, we do still observe jetting, even at higher outer flow rates, i.e., above a threshold $W e_{\mathrm{i}}>7$. The here-observed topology with respect to the outer capillary number is thus qualitatively different from the previous study (Utada et al. 2007). We also observe jetting below a critical $C a_{\mathrm{o}}$. After an analysis of the recorded videos, we can assign this effect and its transition to a suppression of droplet pinch-off due to the limited flow rate of the more viscous outer fluid. For pinch-off to occur, the outer fluid has to flow into the region around the necking jet. In this "confined jetting" regime, the core exhibits significant diameter modulations when leaving the nozzle, but these perturbations do not significantly grow downstream as one might expect in a more open scenario. As one would expect, this breakup delay is more pronounced the smaller the outer fluid flow rate. This confinement effect was indeed identified in previous work and is to be expected for a high injection ratio like the one used here $\sqrt{\frac{A_{\mathrm{i}}}{A_{\mathrm{o}}}}=0.78$ (Guillot et al. 2007; Nunes et al. 2013). At the slowest jet flow rates (i.e., $W e_{\mathrm{i}}<3$ in Fig. 4a), pinch-off can occur downstream-leading to the observed topology of oblong slugs (S). At even lower $C a_{\mathrm{o}}<0.05$, confinement completely inhibits jet breakup, and we observe stable core annular jetting (CA).

For the N4 injection geometry shown in the state diagram of Fig. 4b, the dripping-to-jetting transition as well the transition to more undulant jetting can be readily demarcated and is thus graphically marked with lines, all in analogy to Fig. 4a. While Fig. 4a, b seems similar at first, the attentive reader has recognized that both the dripping regime and the occurrence of jet breakup [i.e., topology slug (S)] are significantly shifted. On the one hand, dripping is now also observed at considerably smaller outer capillary numbers, and on the other hand the jet breakup into slugs $(\mathrm{S})$ occurs at rather high inner Weber numbers $W e_{\mathrm{i}} \approx 10$. The computational fluid dynamics calculations presented to this end will reveal that a variable amount of outer fluid mass and momentum can be transfered through the four side openings (N4) upstream and thus supply additional outer fluid volume available at the pinch-off site to facilitate jet breakup. It is thus important to note that the $\mathrm{N} 4$ geometry effectively reduces the effect of jet confinement, which was predominant in the N0 experiments discussed above. Since this upstream transfer of mass is selfadjusting, we shall see that spontaneous oscillations are now possible in the $\mathrm{N} 4$ geometry.

\subsection{Co-flow modulation by the $\mathrm{N} 4$ injection geometry}

The image analysis of the high-speed videos recorded with the N4 geometry indeed reveals fluid-fluid interface oscillations at the side openings and at the nozzle exit, which are not present with the N0 geometry. Obviously, the upstream transfer of momentum and mass gives rise to a fluid-fluid oscillation. Figure 5 illustrates yet again how the dripping mechanism is distinctively different when comparing the N0 with N4 injection geometry. While the N0 geometry produces a typical Rayleigh-Plateau dripping with the expected necking and droplet pinch-off, the N4 geometry entails close-packed but isolated droplets at the nozzle occurring at significantly higher number rates. The N4 droplets are significantly smaller than the N0 droplets and can exhibit non-spherical shapes (i.e., longitudinal compression with aspect ratio $>1.5$ ); indeed, the $\mathrm{N} 4$ droplets correspond to topological perturbations of wavelengths, which no longer satisfy the first-order Rayleigh-Plateau instability growth argument $\mathrm{kR}_{0}<1$, where $\mathrm{k}$ is the perturbation wavelength and $\mathrm{R}_{0}$ the nozzle aperture radius. In contrast to the $\mathrm{N} 0$ injection geometry, the jet is obviously breaking up inside the N4 nozzle. Further downstream, the combined actions of gravity, entrainment and interfacial tension result in occasional sideshifts and coalescence of these droplets as illustrated in Fig. 5 (right).

To shed light on the underlying physics of the oscillation occurring with the N4 injection geometry, it is instructive to perform a detailed frequency analysis. We have plotted the N4 oscillator frequency as a function of inner and outer fluid velocities, $v_{o}, v_{i}$, in Fig. $6 a, b$, respectively. We note that interface topological oscillations could be readily observed in the videos far into the regimes classified as jetting; however, their amplitudes were too small to cause jet breakup. We can deduce from Fig. 6a that the oscillator frequency exhibits a significant dependence on the outer fluid velocity. Likewise, this frequency analysis allows differentiating between two different oscillator modes, below 


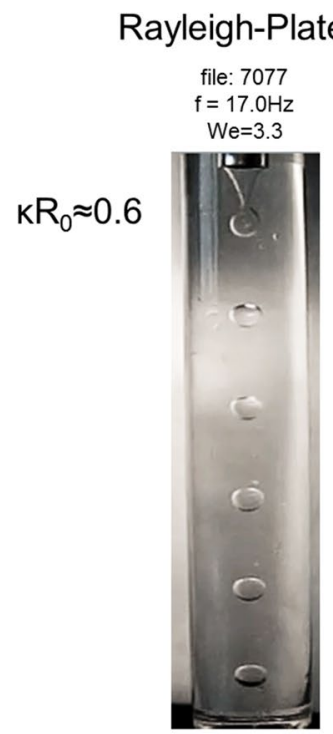

(a)

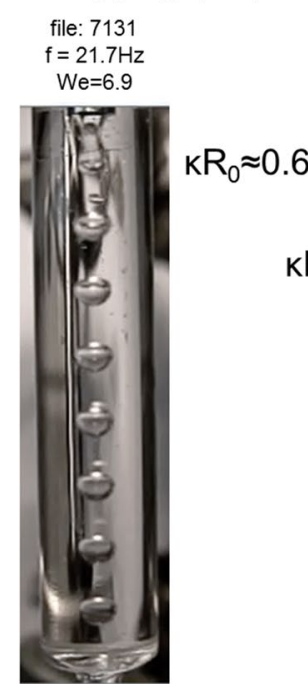

(b)

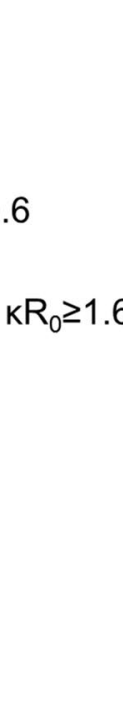

1.6

(c)

$\mathrm{We}=3.6$

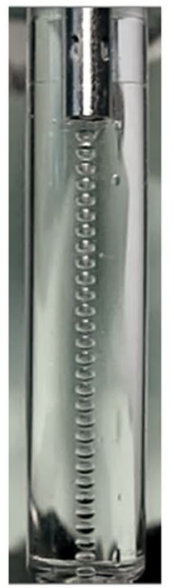

oscillatory dripping (N4)

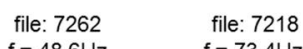

$\mathrm{f}=48.6 \mathrm{~Hz} \quad \mathrm{f}=73.4 \mathrm{~Hz}$

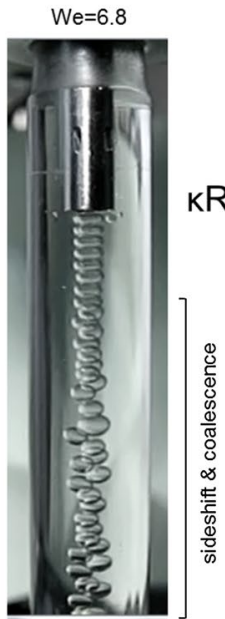

(d)
Fig. 5 Snapshots of co-flow topologies of dripping (D) for two different inner jet velocities $\left(W e_{\mathrm{i}}=3.3\right.$ and $\left.W e_{\mathrm{i}}=6.9\right)$. (Left) with the N0 injection geometry the driving forces for breakup are interfacial tension per jet energy (i.e., $W e_{\mathrm{i}}>1$ ) and entrainment (i.e., $C a_{\mathrm{o}}>1$ ); the pinch-off neck is visible to a characteristic distance away from the capillary tip (a) and the emerging droplets are approximately spheri-

and above the dripping-to-jetting transition. The two modes are termed as mode A and mode B in Fig. 6a. Interestingly, we note also that mode $\mathrm{B}$ has a frequency resembling the third harmonic of mode A (see dashed line in Fig. 6a) but a significantly smaller amplitude at the fluid-fluid interface. Besides, the analysis of the high-speed video recordings reveals how mode $\mathrm{B}$ modulations at the interface vanish downstream and are gradually replaced by longer wavelengths that grow upon the Rayleigh-Plateau breakup mechanism. The measurement point (d) seems to scatter away from the main trend (i.e., mode A). This deviation is not erratic, but the result of an enhanced dependence of dripping frequency to inner fluid velocity, $\mathrm{v}_{\mathrm{i}}$, at higher $\mathrm{v}_{\mathrm{o}}$, which can be readily verified from Fig. 6b. For the reader's convenience, points (c) and (d) in Fig. 6 correspond to the video snapshots depicted previously in Fig. 5.

The N4 oscillator frequency is about an order of magnitude less sensitive to the inner velocity, $v_{\mathrm{i}}$ (Fig. 6b) than it is to the outer fluid velocity, $v_{0}$ (Fig. 6a). The trend with $v_{\mathrm{o}}$ is nevertheless unmistakable and thus optically outlined.

While the dependence of the dripping frequency from the inner flow velocity could be intuitively expected for a conventional injection geometry (Rayleigh-Plateau mechanism), the here-observed even stronger dependence on the outer fluid velocity is characteristic for injection with the N4 oscillator geometry. The role of the outer fluid in exchange of mass and momentum as introduced by the cal (small lateral distortion due to cylinder glass tube optics); the pinch-off frequencies are relatively small near $20 \mathrm{~Hz}$, see (a) and (b), and weakly depending on $v_{\mathrm{i}}$. (Right) oscillatory dripping observed with the N4 injection geometry, exhibiting much higher frequencies of individual droplets exiting the nozzle

upstream side openings shall be further scrutinized using computational simulation.

\subsection{Assessment using computational fluid dynamics}

Using computational fluid dynamics (CFD), we have simulated fluid-fluid co-flow in the two different flow geometries $\mathrm{N} 0$ and N4 over an extended range of fluid velocities. With this tool, it was possible to gain some valuable additional insights into the hidden interface topology and jet breakup inside the injector. The mathematical basis for these computational fluid dynamics calculations is described in Sect. 2.2. Although the simulation does not completely capture all dynamic transitions seen in our experiment, we want to include the CFD results into the discussion here since they allow a better illustration and understanding of the observed flow patterns during oscillations.

Figure 7 displays such a CFD simulation for the N0 and $\mathrm{N} 4$ geometries. The flow velocities were chosen, so that the flow pattern is expected to be near the experimentally observed dripping-to-jetting transition for the NO geometry, but in the oscillating scenario for the $\mathrm{N} 4$ geometry (see Fig. 4). The simulated flow pattern in the NO geometry is indeed predominantly jetting, with appearance of confinement-delayed Rayleigh-Plateau instability breakup. As can be readily seen from the magnified nozzle region with the N4 side openings in Fig. 7, a variable mass transfer of outer 

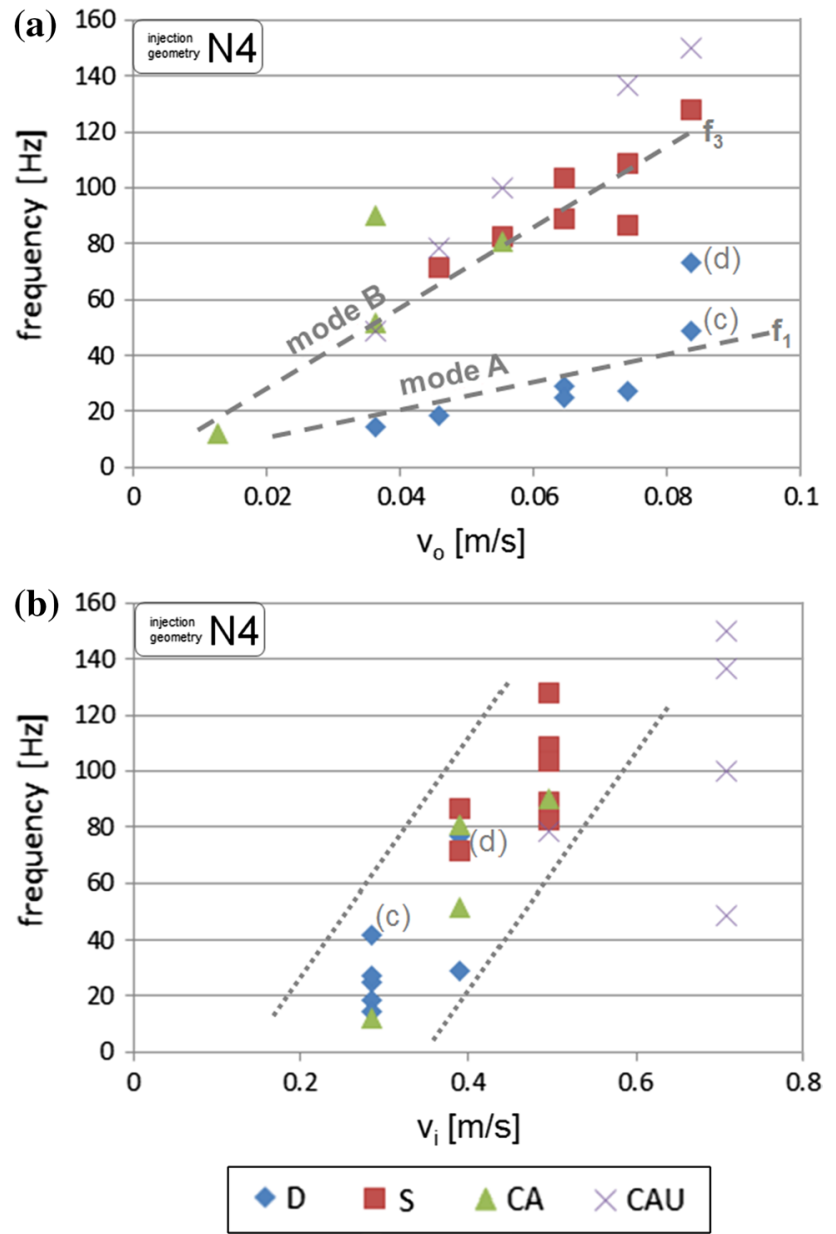

Fig. 6 Frequency of N4 fluid-fluid oscillator as a function of outer fluid velocity, $v_{\mathrm{o}}$, or inner fluid velocity, $v_{\mathrm{i}}$. a The dependence on $v_{\mathrm{o}}$ is pronounced and allows distinction of at least two modes $(A$ and $B)$, whereby mode $B$ seems to be the third harmonic of mode A; for the readers convenience, the gray dashed lines indicate a 1:3 frequency ratio. $\mathbf{b}$ There is a generally positive dependence of the oscillator frequency on $v_{\mathrm{i}}$ albeit at approximately ten times smaller slope than for the dependence on $v_{\mathrm{o}}$; we have added parallel gray dotted lines to highlight this general trend. For undulant jets (CAU), the $v_{\mathrm{i}}$ dependence is less evident. The points marked with $(c)$ and $(d)$ correspond to the situations depicted in Fig. 5

fluid into the inner space occurs. This produces an interface oscillation near the third harmonic in this case. We recognize this internal interface modulation as the basic mechanism of the biphasic fluid-fluid oscillator. At a later time of same CFD simulation, e.g., $t=0.515 \mathrm{~s}$, the flow of outer fluid to the inside nearly vanishes and the oscillation is strongly confined. We attribute the lack of sustained steadystate oscillation in this CFD to the missing gas-spring compliance in the simulation and in the limit of strongly confined jet also to the finite resolution inherent to the hereused volume of fluid (VOF) representation for jet splitting.

The CFD approach allows one to also simulate scenarios that are outside the experimental parameter range. Figure 8

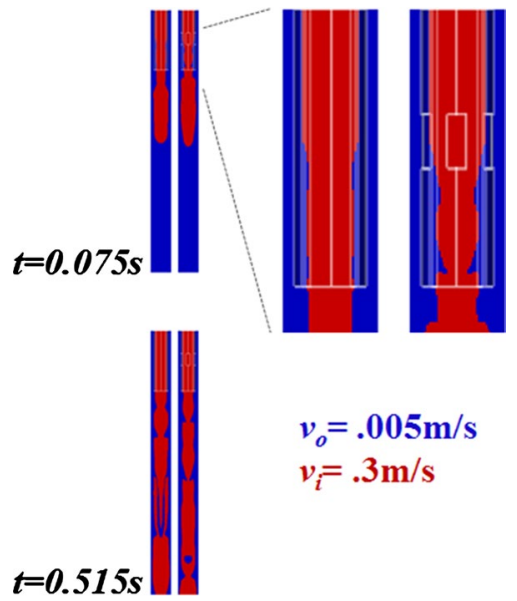

Fig. 7 CFD simulation showing the flow topology at velocities corresponding to inner velocity $v_{\mathrm{i}}=0.3 \mathrm{~m} / \mathrm{s}\left(W e_{\mathrm{i}}=4.08\right)$ and outer velocity $v_{\mathrm{o}}=0.005 \mathrm{~m} / \mathrm{s}\left(C a_{\mathrm{o}}=0.142\right)$ the immiscible fluids are colored red (water, inside) and blue (Vaseline, outside). This corresponds to a situation near the experimentally observed dripping-to-jetting transition outlined in Fig. 4. Two different CFD snapshots at $t=0.075$ and $t=0.515 \mathrm{~s}$ are shown here. The inset is a magnification of the region around the side openings; notably we observe entrainment of outer fluid (blue), which causes a forced oscillation of the fluid-fluid interface inside the nozzle and significant necking near the exit. The magnification allows distinguishing three periods of undulations within the nozzle, which are indicative for a higher harmonic mode of oscillation (color figure online) (a) $v_{0}$

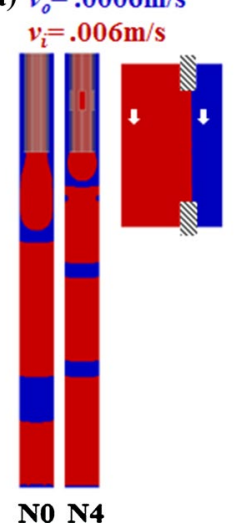

(b) $v_{0}=.008 \mathrm{~m} / \mathrm{s}$ $v_{i}=.003 \mathrm{~m} / \mathrm{s}$

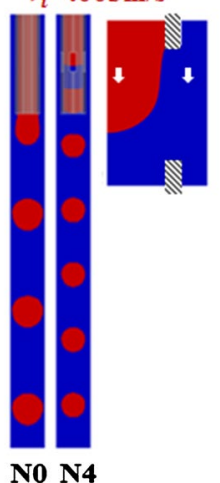

(c) $v_{o}=.08 \mathrm{~m} / \mathrm{s}$

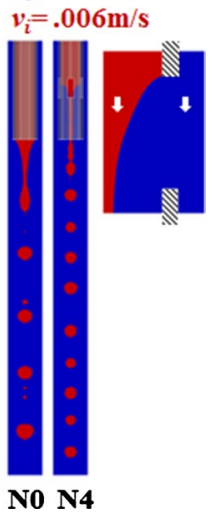

Fig. 8 CFD study of interface topology at three selected flow velocities; each comparing the two co-flow geometries, N0 and N4; the immiscible fluids are colored red (water, inside) and blue (Vaseline, outside); each inset shows a magnified view of the flow pattern around the side openings; situation; while a and $\mathbf{c}$ illustrate extreme examples of outer fluid velocity, $\mathbf{b}$ is a low velocity example, which is archetype for the pinch-off mechanism of the base harmonic oscillation in the $\mathrm{N} 4$ geometry (color figure online)

shows a selection of simulated fluid-fluid topologies obtained for a wide range of fluid velocities. As seen above, a variable amount of viscous outer fluid can enter through the N4 side openings - at a ratio that is related to the relative momentum densities, to say the flow rates, $v_{\mathrm{i}}$ and $v_{\mathrm{o}}$, of the two fluids. 
Figure 8a shows a simulation for ultra-low outer fluid velocity. The inner fluid entirely fills the tube in patches after the nozzle; the jet does break up in fulfillment of the continuum equation. Although this scenario is close to equilibrium and reminiscent of a phase-separation, the noteworthy effect of the N4 injection geometry is to produce smaller phase domains. This effect of the N4 geometry near equilibrium is, however, rather subtle since we could not observe significant transfer of mass at the side openings (see inset).

Figure $8 \mathrm{~b}$ illustrates a complete jet breakup (D) scenario at very low inner flow rates. This scenario was experimentally not accessible due to injector backflow issues. Both injection geometries $\mathrm{N} 0$ and $\mathrm{N} 4$ produce jet breakup driven by surface tension due to the Rayleigh-Plateau instability. However, the N4 geometry permits complete jet breakup yet before leaving the nozzle. The resulting dripping mechanism is characteristic for the N4 geometry, and it yields smaller droplets at higher number frequency; the magnified inset shows how the droplet pinch-off is forced inside the nozzle by variable mass transfer across the side opening. This is the prototype topology of forced dripping at the base harmonic mode of the oscillator, as also observed in the real experiments at small inner and midrange outer flow velocities.

Figure 8c illustrates the dramatic effect of outer fluid entrainment on the inner jet diameter in a case where the outer fluid velocity is even higher, while the inner velocity is kept the same as in Fig. 8a. The strongly entrained central jet is confined and breaks up according to the RayleighPlateau instability mechanism; i.e., smaller jets breakup into smaller droplets because the fastest growing instability amplitude is proportional to the jet diameter. Remarkably, a spatially delayed jet breakup is apparent when the jet is accelerated and entrained inside the capillary as previously reported (Eggers and Villermaux 2008).

\subsection{Alternative injection geometries and resulting interface topologies}

To this point, we have documented the interface topologies produced with the two injection geometries, N0 and N4. In addition to these variants, we have also implemented real fluid experiments using more complex injection geometries. These alternative injection geometries include the use of a different number of side openings, namely N1, N2, $\mathrm{N} 3$ as well as the usage of different baffle plates terminally capping the ends of the injection capillaries (Fig. 9).

Our findings show that the N2, N3 and N4 injection geometries produced qualitatively similar symmetric topologies as described above for the $\mathrm{N} 4$ geometry, whereas only the $\mathrm{N} 1$ geometry was able to produce asymmetric flow patterns. (a)

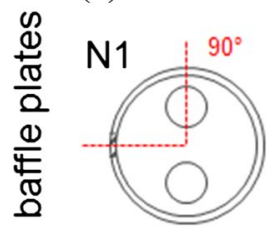

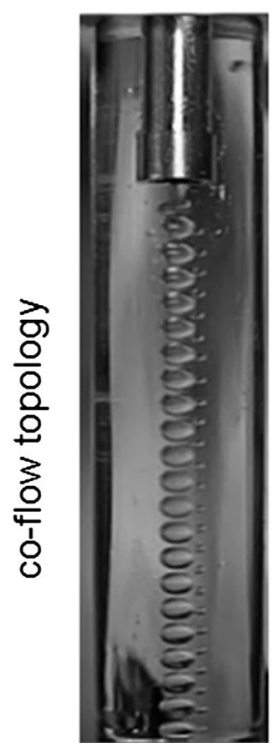

(b)
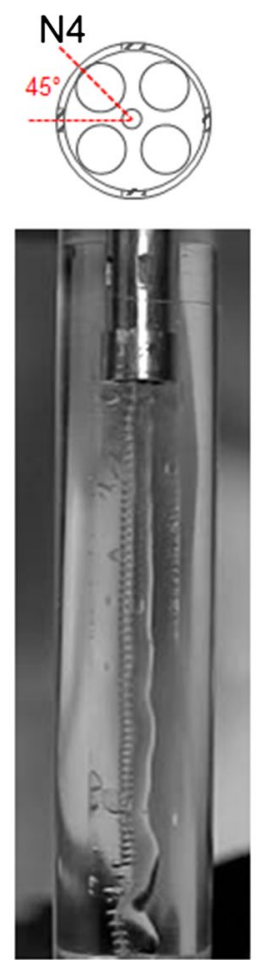

(c)

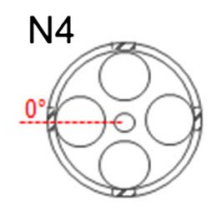

Fig. 9 Flow topologies realized in real flow experiments (water in Vaseline) using alternative injection geometries. The terminal baffle plate layout is schematically shown at the top of each photograph; a asymmetric injection with one side opening, N1, and terminal baffle plate with two circular openings rotated at $90^{\circ}, \mathbf{b}$ symmetric injection with four side openings, N4, and terminal baffle plate with five circular openings at rotational angle $45^{\circ}$, c symmetric injection with four side openings, N4, and terminal baffle plate with five circular openings aligned at $0^{\circ}$

Furthermore, the introduction of a terminal baffle plate allowed for a splitting of the central fluid thread into multiple ones. Thereby, the rotational angle of the baffle plate is relevant for the splitting ratios. In our experiments, the accuracy of manually setting the rotational angle was unfortunately limited to an accuracy of some $\pm 3^{\circ}$. In Fig. 9, we display a selection of three representative interface topologies, which illustrate the relevance of rotational symmetry between side openings and baffle plate openings for the flow ratios of jet splitting.

The flow topology shown in Fig. 9a was realized by means of the N1 injection geometry capped by a baffle plate with two circular openings arranged at a $90^{\circ}$ angle. The asymmetry of this arrangement is reflected in the occurrence of two dripping lines with differently sized droplets. It is obvious that the droplet frequency is higher than expected for a simple Rayleigh-Plateau instability breakup. Also the droplet frequency is independent of droplet size and there is no necking visible at the nozzle. These 
findings confirm that the breakup is indeed produced by the biphasic fluid oscillator formed by one single side opening. The breakup into droplets must occur inside the capillary, i.e., upstream of the baffle plate; these droplets are then split one more time into two unequal droplet threads by the baffle plate-most likely by splitting each droplet individually.

Figure $9 \mathrm{~b}$ conversely illustrates an example of a jet splitup into both a dripping and a jetting thread. Frequency and size of the dripping line are again distinctive for a forced oscillatory breakup in this N4 geometry, excluding simple Rayleigh-Plateau breakup. However, the terminal baffle plate at an angle of $45^{\circ}$ seems to break the symmetry of the N4 base oscillator and change the mode of oscillation. It would indeed be revealing to rotate the baffle plate in small steps to check for different oscillator modes-unfortunately this was not readily possible in our experiment.

The co-flow topology depicted in Fig. 9c suggests that the symmetry of the N4 injection geometry is essentially retained when capped by a baffle plate of identical and aligned fourfold symmetry. It means that the dripping flow is split into multiple threads of comparable magnitude and droplet size.

Our experimental and simulation results suggest that the presented biphasic fluid oscillator can also be understood as a flow-focusing device connected to an oscillating bypass flow-the oscillation being induced by slef-adjusting upstream mass and momentum transfer. A practically relevant question is the maximum available range of tunable frequency, which was less than one order of magnitude in our case. The observed high dependency of the oscillator frequency on the outer fluid velocity, $\mathrm{v}_{\mathrm{o}}$, however strongly suggests that the injection ratio $\sqrt{\frac{A_{\mathrm{i}}}{A_{\mathrm{o}}}}$, which was not varied in this study, could be a practically sensitive parameter to tune the base oscillation frequency, by controlling the degree of jet confinement. In any case, a minimum oscillation amplitude of the upstream mass transfer is indeed needed to provide the peak flow of outer fluid volume into the nozzle, needed to allow complete inner jet breakup at the pinch-off site. Therefore, the absolute flow rates of the two fluids will probably have to be adjusted as well. Further experimental work and simulation may be needed to answer this question and also isolate the role of the gasspring loaded reservoir in the evolution of sustainable oscillations with incompressible model fluids.

Our oscillator model study did not include the effect of different viscosities of inner and outer fluids. Since we were able to link the oscillator behavior with previous dripping studies in terms of dimensionless capillary and Weber numbers, we are confident that within a firm experimental window, the effect of viscosity is as expected from its defined contribution to these dimensionless numbers.
In future, we want to design and build similar microfluidic nozzles for fiber melt-spinning-our starting motivation. For this extension, we must consider nonlinear flow properties of polymer melts, which can give rise to instabilities themselves (Eggers and Villermaux 2008). Such normally undesirable instabilities are due to the energy stored in the viscoelastic polymer melt under pressure (Hatzikiriakos and Migler 2005; Agassant et al. 2006). In the case of a biphasic oscillator, we expect that this specific property of polymer melts may actually take the effect of the gasspring loaded reservoir that was found to be a relevant factor in the here-presented model experiments with otherwise incompressible fluids. In the established melt-spinning process, the spin pump effectively decouples the spinneret flow dynamics from the extrusion part. Yet, the dynamic elasticity of polymer melts may thus locally provide the volume swell necessary to enhance the interface oscillation amplitude and finally allow oscillatory dynamic fluid core pinch-off inside the design-adjustable but limited volume of a bicomponent spinneret.

\section{Conclusions}

In this paper, we present the design basics for a simple passive biphasic fluidic oscillator, which can produce droplets at significantly higher frequency than the commonly used passive Rayleigh-Plateau dripping mechanism. The independence on compliant or actively controlled elements makes this type of oscillator well suited for high-speed or high-temperature microfluidic applications. Mainly owing to jet confinement, the use of a high injection ratio allows significant control of the oscillator frequency by outer fluid flow. Computational fluid dynamics calculations helped identifying the important role of upstream mass and momentum transfer for the excitation of fluid-fluid interface oscillations.

Our results are in line with previous studies, done for more conventional injection geometries, and our analysis of different interfacial topologies in a state diagram confirms the importance of dimensionless Weber and Capillary numbers.

Additionally, we demonstrate the possibility to further expand the variety of attainable topologies by splitting the jet into multiple threads using a terminal baffle plate, while the symmetry of breakup can be controlled by relative rotational symmetry.

Acknowledgments We acknowledge M. Becker and U. Heck from DHCAE Tools GmbH, Krefeld, Germany for their help with the basic setup for the CFD calculations. This project was supported by the Swiss National Science Foundation's Smart Materials Program (NRP-62). 
Open Access This article is distributed under the terms of the Creative Commons Attribution 4.0 International License (http://creativecommons.org/licenses/by/4.0/), which permits unrestricted use, distribution, and reproduction in any medium, provided you give appropriate credit to the original author(s) and the source, provide a link to the Creative Commons license, and indicate if changes were made.

\section{References}

Agassant JF, Arda DR, Combeaud C, Merten A, Munstedt H, Mackley MR, Robert L, Vergnes B (2006) Polymer processing extrusion instabilities and methods for their elimination or minimisation. Int Polym Proc 21(3):239-255

Bannwart AC (2001) Modeling aspects of oil-water core-annular flows. J Petrol Sci Eng 32(2-4):127-143

Castro-Hernández E, Gundabala V, Fernández-Nieves A, Gordillo JM (2009) Scaling the drop size in coflow experiments. New J Phys 11:075021

Cramer C, Fischer P, Windhab EJ (2004) Drop formation in a coflowing ambient fluid. Chem Eng Sci 59(15):3045-3058

Deshpande S, Anumolu L, Trujillo M (2012) Evaluating the performance of the two-phase flow solver interFoam. Comput Sci Dis$\operatorname{cov} 5(1): 014016$

Devaraju NSGK, Unger MA (2012) Pressure driven digital logic in PDMS based microfluidic devices fabricated by multilayer soft lithography. Lab Chip 12(22):4809-4815

Eggers J, Villermaux E (2008) Physics of liquid jets. Rep Prog Phys 71(3):036601

Ghosh S, Mandal TK, Das G, Das PK (2009) Review of oil water core annular flow. Renew Sustain Energy Rev 13(8):1957-1965

Guillot P, Colin A, Utada AS, Ajdari A (2007) Stability of a jet in confined pressure-driven biphasic flows at low reynolds numbers. Phys Rev Lett 99(10):104502-1-104502-4

Gupta A, Matharoo HS, Makkar D, Kumar R (2014) Droplet formation via squeezing mechanism in a microfluidic flow-focusing device. Comput Fluids 100:218-226

Hatzikiriakos SG, Migler KB (2005) Polymer processing instabilitiescontrol and understanding. Marcel Dekker, New York

Higuera P (2013a) Realistic wave generation and active wave absorption for Navier-Stokes models: application to OpenFOAM ${ }^{\circledR}$. Coast Eng 71:102

Higuera P (2013b) Simulating coastal engineering processes with OpenFOAM $^{\circledR}$. Coast Eng 71:119

Hooper AP, Boyd WGC (1983) Shear-flow instability at the interface between two viscous fluids. J Fluid Mech 128:507-528

Joseph DD, Bai R, Chen KP, Renardy YY (1997) Core-annular flows. Annu Rev Fluid Mech 29:65-90

Kim SJ, Yokokawa R, Takayama S (2013) Microfluidic oscillators with widely tunable periods. Lab Chip 13(8):1644-1648

Kouris C, Tsamopoulos J (2001) Dynamics of axisymmetric coreannular flow in a straight tube. I. The more viscous fluid in the core, bamboo waves. Phys Fluids 13(4):841-858

Kouris C, Tsamopoulos J (2002) Dynamics of the axisymmetric coreannular flow. II. The less viscous fluid in the core, saw tooth waves. Phys Fluids 14(3):1011-1029

Meister BJ, Scheele GF (1967) Generalized solution of the tomotika stability analysis for a cylindrical jet. AIChE J 13(4):682-688
Meister BJ, Scheele GF (1969a) Drop formation from cylindrical jets in immiscible liquid systems. AIChE J 15(5):700-706

Meister BJ, Scheele GF (1969b) Prediction of jet length in immiscible liquid systems. AIChE J 15(5):689-699

Mosadegh B, Kuo CH, Tung YC, Torisawa YS, Bersano-Begey T, Tavana H, Takayama S (2010) Integrated elastomeric components for autonomous regulation of sequential and oscillatory flow switching in microfluidic devices. Nat Phys 6(6):433-437

Nguyen TV, Duncan PN, Ahrar S, Hui EE (2012) Semi-autonomous liquid handling via on-chip pneumatic digital logic. Lab Chip 12(20):3991-3994

Nightingale AM, Phillips TW, Bannock JH, de Mello JC (2014) Controlled multistep synthesis in a three-phase droplet reactor. Nat Commun 5:1-8

Nunes JK, Tsai SSH, Wan J, Stone HA (2013) Dripping and jetting in microfluidic multiphase flows applied to particle and fibre synthesis. J Phys D-Appl Phys 46(11):114002-1-114002-20

Plateau J (1873) Statique Expérimentale et Théorique des Liquides Soumis aux Seules Forces Moléculaires. Gauthier-Villars, Paris

Rakszewska A, Tel J, Chokkalingam V, Huck WTS (2014) One drop at a time: toward droplet microfluidics as a versatile tool for single-cell analysis. Npg Asia Mater 6

Rayleigh L (1879) On the Capillary Phenomena of Jets. Proc R Soc Lond 29(196-199):71-97

Richards JR, Beris AN, Lenhoff AM (1993) Steady laminar flow of liquid-liquid jets at high Reynolds numbers@f. Phys Fluids A 5(7):1703-1717

Richards JR, Beris AN, Lenhoff AM (1994) Dynamic breakup of liquid-liquid jets. Phys Fluids 6(8):2640-2655

Richards JR, Beris AN, Lenhoff AM (1995) Drop formation in liquid-liquid systems before and after jetting. Phys Fluids 7(11):2617-2630

Rosenfeld L, Lin T, Derda R, Tang SKY (2014) Review and analysis of performance metrics of droplet microfluidics systems. Microfluid Nanofluid 16(5):921-939

Scheele GF, Meister BJ (1968) Drop formation at low velocities in liquid-liquid systems: Part I. Prediction of drop volume. AIChE J 14(1):9-15

Tomotika S (1935) On the instability of a cylindrical thread of a viscous liquid surrounded by another viscous fluid. Proc R Soc Lond A Math Phys Sci 150(870):322-337

Utada AS, Fernandez-Nieves A, Stone HA, Weitz DA (2007) Dripping to jetting transitions in coflowing liquid streams. Phys Rev Lett 99(9):094502-1-094502-4

Utada AS, Fernandez-Nieves A, Gordillo JM, Weitz DA (2008) Absolute instability of a liquid jet in a coflowing stream. Phys Rev Lett 100(1):014502-1-014502-4

Vempati B, Panchagnula MV, Öztekin A, Neti S (2007) Numerical investigation of liquid-liquid coaxial flows. J Fluids Eng Trans ASME 129(6):713-719

Vempati B, Panchagnula MV, Öztekin A, Neti S (2010) Combined buoyancy and viscous effects in liquid-liquid flows in a vertical pipe. Acta Mech 210(1-2):1-12

Xia HM, Wang ZP, Nguyen VB, Ng SH, Wang W, Leong FY, Le DV (2010) Analyzing the transition pressure and viscosity limit of a hydroelastic microfluidic oscillator. Appl Phys Lett 104(024101):1-4 